\title{
APPROXIMATIONS, IDEALIZATIONS, AND MODELS IN STATISTICAL MECHANICS $\dagger$
}

\author{
Chuang Liu
}

1. Introduction. The overall structure of the paper's main argument goes as follows. The existing theories of approximation and idealization are found to be inadequate because they oversimplify the measure of approximation and misidentify the purpose (and therefore the measure) of idealization, respectively. It is then argued that to idealize is not necessarily to postulate conditions which are themselves approximate (or realistic) or whose gradual removal guarantees asymptotically more approximate results; it is also -- mainly (I argue) -- to set up theoretical models so that approximate as well as true laws of nature can be either applied (if they are already known) or discovered (if they are not yet known). Because of this close connection with model-building in science and because of the extreme scope and complexity of the activity, it does not seem plausible that there is a logical model for idealization -- something analogous to Hempel's D-N model for explanation. Rather, I content that to understand the rich content of this notion, one must conduct detailed analysis of its different guises in the praxis of science. Thermostatistical physics is chosen here because it contains the oldest and best understood microscopic accounts of macroscopic behavior; it is where a clear divide between the phenomenological and the fundamental laws shows up in its simplest form; and it is fundamental in that without it, we cannot even understand why there is 'anything' (i.e. observable macro-objects) rather than 'nothing' (i.e. unobservable micro-objects). My hope is to unearth some important aspects of idealization through a detailed analysis of thermostatistical models.

2. Idealization and approximation. According to the traditional characterization, an idealized law (sentence) ${ }^{1}$ or theory is intuitively understood as one that is true under the ideal conditions which differ from the actual conditions either slightly -- so that the law is approximately true -- or significantly but, when the differences are systematically reduced, the law becomes more and more approximate. And a sentence is approximately true if it is in fact false but very close to being true (cf. Barr 1971, 1974). There are mainly two kinds of theories for approximate truth: the state-space and the possible-world theory. According to the most comprehensive state-space theory (Niiniluoto 1984: ch.7, 19862 , see also Marquis 1991), the approximation to truth is measured by the metric distance between two cells (discrete space) or points (continuous space) on an Euclidean space of the states of 
the world. Let $S$ be a predicate space which fully describes all the possible states of the world by having $n$ families of one-place, mutually orthogonal predicates (i.e. $S$ is spanned by n axises). The distance between any two cells in an $S$-space is the usual Euclidean measure: $D=\sqrt{\sum_{i} d_{i}^{2}}$, where $d_{i}$ is the difference between the two cells on the ith axis. (This scheme can be extended to the continuous case and to the case for generalizations or laws (cf. Niiniluoto 1986). ) And then, one needs to specify the limit of approximation (a function of $D$ ) within which all sentences are considered approximately true. One should note that this is a theory of what approximation is, not one of how we come to know what is, and what is not, approximately true.

The possible-world theory (Hilpinen 1976; Weston 1987, 1992) runs roughly as follows. Let us think of a possible world, $w$, (or a possible way the world might be) as a possible model. Then we have the following.

A sentence $\varphi$ is approximately true (under $\Sigma$ ) in $w$ if, and only if, there is another world, $w^{\prime}$, such that it is $\alpha$-close to $w$ and satisfies $\varphi$,

The central element of this theory is the measure of similarity or closeness -- $\Sigma$-- between possible models (or worlds). Hilpinen (1976) follows Lewis in trying to articulate the notion of similarity among possible worlds, but comes short of offering a quantitative measure for it -- the difficulty is carefully explained by Lewis $(1986)$, while Weston's $(1987,1992)$ construal of $\Sigma$ is very similar to $D$ in the state-space theory.

Then, the notion of idealization can also be made precise, and there are mainly two traditional theories for it. For one, we mainly follow Laymon's (1980, 1985, 1989) comprehensive theory of confirmation, of which idealization is a central component. Given a generic theory $T$, and a set of $n$ ideal-condition claims: $I=\left\{I_{i} \mid i=1, \ldots, n\right\}$, such that $I_{j}$ is less idealized (or more realistic) than $I_{i}$, where $j>i$, we have that for all $i \in\{1, \ldots, n\}, I_{i}: T \Rightarrow T_{i}$, and $T_{i} \mid=P_{i}$, where $P=\left\{P_{i} \mid i=1, \ldots, n\right\}$ is a set of predictions. Suppose that the non-idealized theory, $T^{U}$, predicts the true $P^{U}$; and let $\Delta^{-} I=\left|I_{j}-I_{i}\right|$ and $\Delta^{+} P=\left|P_{j}-P_{i}\right|$ (where $j>i$ ) denote respectively the factual distance between two ideal conditions and predicted states of affairs, we then have,

[A] $T$ is a good idealized theory (or the idealization is justified) if it is always true that $T: \Delta^{-} I \Rightarrow \Delta^{+} P$; otherwise, it is not (or the idealization is not justified). 
In other words, when $I \rightarrow 0$, we have $T \rightarrow T^{U}$ and $P \rightarrow P^{U}$, if $T$ is a good idealized theory. I suppose that one can likewise define a rate of convergence for idealizations of the same kind -- such as $\gamma=-\Delta^{+} P / \Delta^{-} I$, when $\Delta^{-} I \rightarrow 0-$ and claim that one idealization in the set is better than another if its rate of convergence is higher than that of the other. The other theory is Nowak's $(1972,1980)$, which, as shown for instance in Liu 1999a, can be understood as a special case of Laymon's theory, if some slight modifications are made. ${ }^{3}$

2.1. The main problems. Suppose that we follow the possible-world theory of approximation, which is equipped with a clearly formulated sense of approximation, $\Sigma$. The space of possible worlds (or models) is supposed to be Euclidean. This implies that when one says, for instance, that sentence $A$ is more approximate than sentence $B$, given that they are comparable (e.g. they are about a person's height), one means that the total factual distance between $A$ and the truth is smaller than that between $B$ and the truth. The total factual distance depends not only on the magnitude of each individual distance but also on the number of such distances; and a factual distance is a magnitude that is easy to define between certain types of states but difficult for other types. It is safe to assume that as long as one is dealing with quantitative theories in which possible states of affairs are determined by the values of the main variables (i.e. degrees of freedom), a factual distance is simply defined as the Euclidean distance between two sets of such values.

However, this is not true for many such spaces. The point can be easily understood by imagining a space partitioned by laws of nature which prohibit the crossing from one region to another. For example, a phase space for particles may be divided into two regions : in one, particles move with speeds less than $c$ (the speed of light) -- i.e. ordinary objects -- and in the other, with speeds greater than $c$-- i.e. tachyons. Suppose that a particle moves at a speed very close to, but less than, $c$. To attribute any speed, $v<c$, to it would be a better approximation than giving it a speed slightly over $c$. But according to the Euclidean metric, the latter is factually closer to the truth than most of the former, and so the latter should be more approximate, which is clearly the wrong judgment.

One may perhaps hope that the problem can be avoided by identifying fault lines in each case and construct multiple $\Sigma$ 's accordingly for the space. However, the matter does not seem so simple, even if it is taken as a metaphysical -- rather than an epistemological -- problem. The space of either possible worlds or possible states of affairs is very much unlike the $p V T$ (pressure-volumetemperature) phase space of thermal systems, where one has mostly homogeneous pure phases except 
a few lines of phase transitions near which the systems' behavior is singular (more on this later). To see this point, let us consider again our example with the speed of light. With regard to particles that are not tachyons, suppose that it is true that a particle, $\alpha$, is moving with a speed $v$ that is less than $c$ but quite close. There are two statements, one says that $\alpha$ moves with a speed $v^{\prime}$ and the other says that it moves with a speed $v^{\prime \prime}$, where $v^{\prime} \in(c, v)$ and $v^{\prime \prime} \in(v, 0)$, both measured in the same reference frame of $v$; and moreover, $\left|v-v^{\prime}\right|<\left|v-v^{\prime \prime}\right|$. Which statement is more approximate then? If the space of possible states of $\alpha$ below $c$ is Euclidean, the statement with $v^{\prime}$ should be regarded as more approximate than the one with $v^{\prime \prime}$; but the opposite seems more reasonable because a particle is more likely to be moving at $v^{\prime \prime}$ than at $v^{\prime}$, considering the difficulty with which a particle has to be accelerated to a speed of $v^{\prime}$.

What the example shows is in fact something general about such spaces of possible worlds or states of affairs: the measure for approximation that can be adequately put on any region of such a space must be a weighted measure, taking into account of the likelihood of each point in the region. This means that measures such as, $D=\sqrt{\sum_{i} d_{i}^{2}}$, covering the whole or most of the space are fundamentally misconceived, for it assumes that all states are equally likely to obtain. This is clearly the exception rather than the rule. Now, perhaps it is possible to incorporate the weights into the measures, but we know neither whether it is indeed possible nor, if it is, how to begin to give even a sketch. Therefore, a general theory of approximation which contains a plausible scheme of evaluating competing claims of approximation remains for now at least a daunting task. ${ }^{4}$

Now, a problem for the traditional theory of idealization. It concerns idealized laws, which are false because of being idealized but which still approximate the true laws. Let us represent an idealized law hypothesis as: $L$ given $I$, where, $I$ is a set of ideal conditions, $L$ is in the form of ' $N(F, G)^{\prime}$, where $N$ denotes natural necessity. ${ }^{6}$ According to the theory of idealization given above, we should say that a law is approximately true if, and only if, either all of $L$ 's factual consequences (e.g. instantiations) are approximately true under $I$ or their approximation is asymptotically improved when $I$ is gradually removed. But here a problem arises. Without any lost of generality and in connection with our later discussion of thermostatistical models, let us suppose that our true law is the isotherm, $L$, of a system in which a phase transition takes place (see Figure 1), and that $L_{1}$ and $L_{2}$ are two laws which are true under ideal conditions, $I_{1}$ and $I_{2}$. 


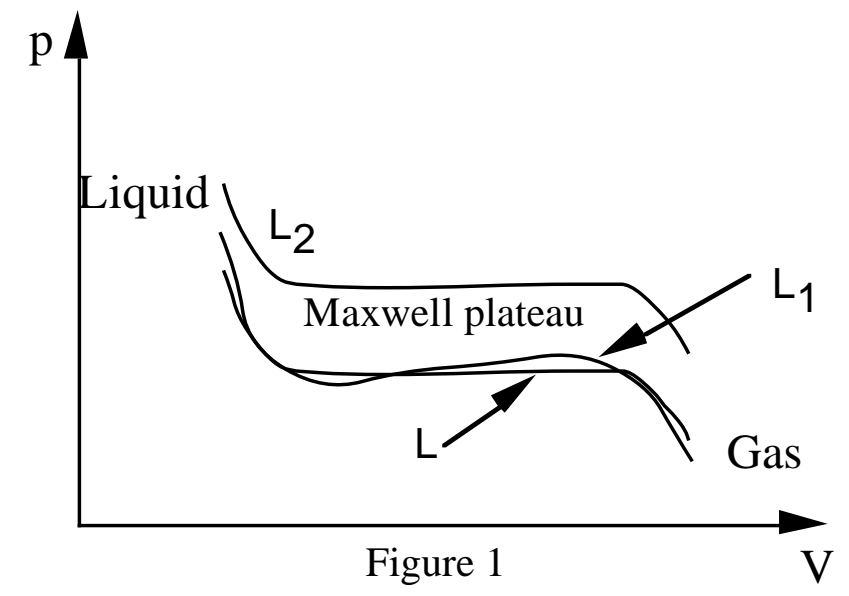

Which of $L_{1}$ and $L_{2}$ is more approximate, given $L$ obtains? If we, by following the traditional theory, compare their factual consequences (i.e. point-wise distances), $L_{1}$ is closer to $L$ than $L_{2}$, but we can clearly see that as a matter of law, it is the opposite: $L_{2}$ at least has singularities in it (i.e. the points at the two ends of the Maxwell plateau), whereas $L_{1}$ is all smooth -- which as we will see in a moment means that there is no phase transition in $L_{1}$ ! And, worse still, part of $L_{1}$ implies that $\partial \mathrm{p} / \partial \mathrm{V}$ $>0$, namely, the pressure increases while the system expands at a constant $T$ ! Similarly, we would say that holding $I_{2}$ is a better idealization than holding $I_{1}$, and yet $I_{1}$ defines a factually closer world (or state) to $L$ than $I_{2}$ does. $L_{1}$ is what Pauli is said to have called a 'not even false' law hypothesis, which in our context must mean that the hypothesis totally misses the form of the intended law. One could always improve the approximation -- the empirical fit -- of such a hypothesis, but should one? But why shouldn't one, if the traditional theory of idealization is correct?

Nor would it help -- for very similar reasons -- by requiring asymptotic improvement of approximation when the ideal conditions are gradually removed.

We now see that there are two dimensions for evaluating idealized laws: factual closeness and form closeness (resemblance). The traditional theory only has the first dimension, which is why it fails.

2.2. A theory of idealization. The reason for the failure of the traditional theories is that there are different types of idealizations, of which approximation production is only one type -- e.g. those idealizations one depends on in finding generalizations in a collection of experimental data. But this is not the same kind of reasoning as the one which assumes that the universe contains only the sun and the earth or is without electromagnetic field. Nor are both the same type as assuming the ratio of an object's speed to that of light is zero or taking the volume of a thermo-system to infinity (i.e. taking the thermodynamic limit, as will be explained later). Nor are they the same as the construction of 
lattice models for bulk matter and for quantum fields. From the perspective of approximation production, we simply do not see anything in common in these different acts of idealizations. We must therefore begin afresh.

Within the scope of science it seems that acts of idealization serve the purpose of 'inventing' or 'isolating' models in which laws of nature can be found (if not known) and/or applied (if known) (see McMullin 1985; Cartwright 1989). The complexity and variability of natural phenomena and our desire to explain them with a small number of simple and invariant laws demand that we disentangle them. The best way of achieving that is to conceptually imagine or design a setting (not obtained in the actual world) in which systems operate in simple and uniform manners so that approximate or true laws can be discovered and (in some cases) tested; and this is what idealizations are primarily used for. (One should not therefore miss the close connection between idealization and thought experiments.) It is only when the actual world with its complex phenomena is thus disentangled into separate theoretical models by idealization is it hopeful that genuine laws of nature may be discovered or applied. Some laws so discovered and tested in one context (or discipline) are not directly applicable in another, even if they are universally true. Again, phenomena in the latter context (or discipline) need to be disentangled, and idealized models need to be set up for the detached components, before the laws become applicable again. Can mechanical laws of motion for Newtonian particles be used to study the phenomena of heat if idealized models are not allowed?

A particular form of this idea is perhaps first clearly articulated by J.S. Mill in Bk. VI of (Mill 1874), namely, genuine laws of moral science can never be discovered by looking for regularities in the observed social events, for though the regularities may be close to the observed facts (i.e. approximately true), they can never be universal enough to qualify for laws. Instead, one should think of social phenomena -- similar to chemical phenomena -- as results of complex combinations of simple systems or processes which obey simple and universal laws -- á la chemistry (see also Cartwright 1997; Wimsatt 1987, 1994; Morrison 1997). The more complex and/or variable the phenomena and the deeper the posited mechanisms of which the laws are to be formulated, the wider the gap between the laws and the observable facts. And the gap should not affect the truth of the laws or theories in question. If one can conceptually (and to some extent even experimentally) cut the world apart at its joints, one can better understand how it actually operates -- i.e. giving explanations -- and predict how not yet existing phenomena may occur (out of a composition of causes which obey the laws). 
The same point can be made from a different perspective. We humans are situated in nature in such a way that some of its component strands -- e.g. regular macroscopic processes -- are made obvious to us. Little art of idealization was required for the ancients to formulate a precise and mathematically sophisticated science of astronomy. That is because the relevant characteristics were clearly revealed to the astronomers by nature -- i.e. by making the sizes of the heavenly bodies and their observers much smaller than the distances that separate them. No such luck existed, for example, for the science of mechanics: few if any surfaces at Galileo's time could be made smooth enough to allow an easy recognition of the separation between motion and friction. It required Galileo's ingenious idealization (cf. McMullin 1985; Suárez 1999) -- which made such a separation -to set the foundation for the discovery of the law of motion. To include, for instance, friction -which de facto accompanies all macroscopic motions in nature -- in the description of a body's state of motion would be to entirely miss a mechanical joint of nature.

What are joints of nature? It is not a question that admits a simple general answer. First, they are supposed to be places in natural processes where component systems or processes meet to produce observable phenomena. Hence, the important things are not the joints but what are, at least conceptually, detachable from the joints. What are these? Are they the same as natural kinds? I do not think they are, but the terms that refer to such detachable parts are similar to natural kind terms. As natural kind terms, they are not absolute but may come and go in the history of science. One of the results of a major scientific revolution is certainly the disappearance of some old terms for detachable parts and the introduction of some new ones. But the concept of such parts -- the most important of them are the fundamental ones -- only makes sense if one accepts the metaphysical view that nature is somewhat like a machine whose working is the result of the combined working of its intricate parts; and different combinations of the same, or of different, parts usually result in different natural processes. They are obviously incompatible with a radically holistic view of nature. Again as natural kind terms, it is up to science to find out which are detachable components in nature and which are not. If there are detachable parts, there must be joints; and the combined actions of the parts through the joints produce the variety of phenomena we observe. If one is a realist about the joints and the parts they connect, one believes that the progress of science is in part a search for the real joints and, hence, parts of nature, while if one is an empiricist, one may believe that the joints and the parts they connect may be purely theoretical constructions, whose justification lies in how well they save the phenomena and satisfy the various methodological criteria of theory selection. 
What exactly is the relation between idealized models and laws? Idealized models are needed for discovering or applying laws in systems or situations other than those in which they are known to hold. ${ }^{7}$ These are true especially for fundamental laws at the microscopic level. It is no exaggeration to say that laws of nature often express exact and true relations of physical necessities among properties of systems which themselves do not exist in the actual world, e.g. frictionless planes. In the context of law discoveries, the acts of detachment, or disentanglement, I mentioned before are often needed to separate the different elementary components or causal processes; and in the context of law applications, one needs to detach the different components of a complex system so that laws which obviously apply to them separately can be tried out on the system to see whether the results of their combined application matches the system's observed (or observable) behavior. The model of a frictionless region, for instance, is a state of the world in which that the region of space in question has nothing inside (except the testing object); and a process in which both electromagnetic and gravitational forces are present can be disentangled into two model processes each of which has one force alone present, other aspects of the processes being the same. We will see in a moment when we discuss thermostatistical models some subtle complications in this way of seeing model-building in science (see also Herfel et al 1995). But as will be shown, the general conception given here is still sound.

What exactly are models in our approach? Are they the same type of models that logicians use? Opinions are divided on this issue in the literature on scientific theories. People such as van Fraassen (1989) and Suppe (1989) (see also Giere 1988) believe that they are the same, while people such as Cartwright (1999) and Morrison (1999) think that they are not. ${ }^{8}$ According to the latter, models are mediators between theories and reality (or phenomena). Here I suggest that the two views can be reconciled. A model can be defined by all the sentences that are true in it or it can be defined independently of these sentences. The set of all real numbers, for instance, can be defined independently of many arithmetic statements that are true of it; and yet it is a model for all such statements and more. The same can be said -- though not as precisely -- of a scientific model that either van Fraassen or Cartwright may have in mind. The ideal gas model is a relational structure in the general sense that it defines a possible world -- one in which the molecules are sizeless and forceless -- and it can be, and is, specified independently of any laws that are true in it. Cartwright et al are right to say that such models are not the theories themselves since a theory consists in part of law-statements, e.g. Boyle's law for the ideal gas model, but van Fraassen et al are also correct since 
models such as the ideal gas model are indeed a possible structure (or a family of possible structures) in which laws such as Boyle's law are true.

How can laws which are true in an idealized model also be true simpliciter? Cartwright $(1983,1989)$ argued that they are not, but I disagree. ${ }^{9}$ Here is a rough sketch of the main reason. Given there are laws, $N(F, G)$, where $F$ and $G$ refer to dispositional properties (or propensities), such laws may hold universally even though one needs an idealized model to display their operations in their pure form. To know how exactly electromagnetic force operates in its pure form, one has to have a system in which only the electromagnetic force acts; but there are in fact no such systems -hence it is an idealized model. But it does not mean that when there are other forces operating -- what actually happens -- the law of electromagnetism fails. As a law relating dispositional properties, it holds at every instant of its presence anywhere and anytime. In the philosophical literature of laws of nature (Dretske 1977; Armstrong 1983, 1978; Lewis 1973,1983; Carroll 1994), dispositional properties are suspect and therefore not included among the legitimate properties relatable by laws. However, if one has any knowledge of physical sciences, one cannot avoid noticing that there the majority of laws are laws relating dispositional properties. For instance, all laws expressed in the form of differential equations are laws of this kind. The law of motion in e.g. Newtonian mechanics is represented by the differential equation relating the second-order time-derivative of a position function and the first-order space-derivative of a potential, not the infinite number and varieties of trajectories as the solutions of the equation under different initial/boundary conditions. The categorists (see e.g. references cited above) cannot even claim in this case that the law of motion comprises the set of all possible solutions of the equation of motion, because then the law is neither definable nor knowable.

However, not all laws are like these. There are plenty of laws in physics or elsewhere that are false but true only in their tailor-made models. Boyle's law of ideal gas is a good example; and so are the mean-field models which we shall see in a moment. And all those laws which are regarded as 'limiting cases' of the more general true laws -- e.g. all the laws in non-quantum and/or nonrelativistic theories -- are also of this kind. We may call them either 'approximate' or 'idealized' laws (cf. Kyburg 1990, ch.10). My point above was simply that it is not necessarily the case that idealized models only produce idealized laws. ${ }^{10}$ And the examples I used above are all true laws in idealized models. ${ }^{11}$

By now it seems apparent that whatever an alternative theory of idealization may be, it will not comprise a set of adequacy conditions and a simple measure for its degrees. However, two concepts 
(or dimensions) seem crucial to the measure of idealizations: detachability and levels of stability. Both are indispensable for the correct and effective description (and understanding) of nature. What we want to achieve by idealizations is really to create models with the right amount of detachment and/or separation of levels. For the former, we see ample examples in the discoveries or applications of simple and universal causal laws in the face of complex phenomena, which by hypothesis are the result of the coïnstantiation of more than one of such causal laws. And for the latter, examples in thermostatistical physics or material science provide excellent illustration.

\section{Idealizations in theories of phase transitions (PT) and critical phenomena (CP).} The theory which describes and explains PT and CP is part of thermostatistical physics, which is usually divided into thermodynamics and statistical mechanics. The theory for PT and CP consists of three different levels, on which different types of models are used. On the first (phenomenological) level, PT and CP are described in pure thermodynamic terms (Stanley 1971; Toda, et al 1983). The most discussed PTs and CPs are two: those of fluid, such as the liquid-gas transition and its critical region, and those of magnet, such as the paramagnetic-ferromagnetic transition and its critical region. Although being entirely different phenomena, they are remarkably similar with respect to PT and CP. It turns out that at the transition from one phase to another below the critical temperature, $T_{c}$, the isotherms develop singularities (i.e. non-differentiable points) ${ }^{12}$; and as $T \rightarrow T_{c}$, all relevant thermodynamic functions diverge in the form of a power law: $F(t) \propto|t|^{-y}$, where $t=\left(T-T_{c}\right) / T_{c}$ and $y=\alpha$ or $\beta$ or $v$ is the critical exponent (Fisher 1974). The respective critical exponents of fluid or magnet have the same values, which is known as the universality of $\mathrm{CP}$ that has no explanation on this first level.

To explain the first-level phenomena, two types of models have been developed. On the second (semi-microscopic) level, we have the mean-field models, which include the van de Waals (dvW) model for fluid and the Weiss model for ferromagnet (cf. Stanley 1971; Goldenfeld 1992). The vdW law (or equation of state) for the 'real' gas, $\left[p+a / v^{2}\right][v-b]=k T$, is an improvement of the ideal gas law, where $a$ and $b$ are introduced to capture for each substance the effect of (i) the attractive interaction between molecules and (ii) the hard-core of each molecule. From this law one is able to derive the critical values: $\mathrm{T}_{\mathrm{c}}, \mathrm{p}_{\mathrm{c}}$, and $\mathrm{V}_{\mathrm{c}}$, and thus the law of corresponding states: $\left[\tilde{p}+3 / \tilde{v}^{2}\right][\tilde{v}-1]=8 \tilde{T}$ (where for any $\mathrm{x}, \tilde{x}=x / x_{c}$ ), an equation free of the explicit presence of $a$ and $b$ and so universally valid for all substances. The 'wiggles' in the isotherms at $T<T_{c}$ are made by Maxwell (1875) into the Maxwell plateaus (see Figure 1), at the two ends of each of which are the 
needed singularities that signal a PT (cf. Emch \& Liu, 2001, sect. 11.3). Despite being a crude model from a microscopic perspective, the vdW model -- and the law that is true in it -- can already do a great deal. As mentioned above, the law of corresponding states shows already the universal nature of thermodynamic properties. For PT and CP, we have mentioned that with the Maxwell plateaus, the PT for fluid are accurately accounted for, and one can use the law to obtain critical exponents. The exponents however disagree with the experimental values, e.g. while the experimental value of $\beta$ is $1 / 3$, the vdW law predicts that $\beta=1 / 2$; but they preserve some experimentally established relations among these exponents.

The Weiss model is designed to explain ferromagnetism. According to this model, the magnet consists of dipoles as tiny compasses which are not only influenced by the external field, $B$, but also by one another, the latter of which is expressed by $I$-- the 'mean-field' created by the rest of the dipoles in the magnet (or part of it). With such a model, one can do a simple substitution: $B \rightarrow B+\alpha I$, in the equations -- more precisely, in the Hamiltonian -- for a paramagnet (i.e. magnets with no PT) and derive ferromagnetism. The Weiss model is a microscopic model without the details of the micro-structure of the magnet. It succeeds in explaining the PT but fails drastically with the estimates of the critical exponents. Moreover, both the vdW model and the Weiss model predict that there are PTs even if the system is only 1-dimensional, which is deemed incorrect when the Isingtype models are used.

The simplest and genuinely microscopic models (at the third level) are the Ising-type models (Emch 1971; Toda et al 1983; Goldenfeld 1992). All models of this type have the following ingredients:

(i) a lattice of $N$ fixed sites of equal distance, and each site is occupied by a spin-1/2 particle that can be in one of two possible states: spin-up $\left(\sigma_{i}=+1\right)$ and spin-down $\left(\sigma_{i}=-1\right)$;

(ii) an interaction between any two sites are given by the term, $J_{i j} \sigma_{i} \sigma_{j}$, where, $i$ or $j=1,2, \ldots, N$, and $J_{i j}>0$ for specified sites (e.g. nearest neighboring sites) and $J_{i j}=0$ otherwise.

The Hamiltonian of the system is therefore written as

$$
H_{N}=-\sum_{<i, j>} J_{i j} \sigma_{i} \sigma_{j}-B \sum_{i} \sigma_{i}
$$

where $B$ is the external field and $\langle i, j\rangle$ means pairs of sites with specified relations only. According to statistical mechanics (cf. e.g. Sklar 1993, ch.2), the partition function, 


$$
Z_{N}=\sum_{\left[\sigma_{1}\right]} \ldots \sum_{\left[\sigma_{N}\right]} \exp \left(-H_{N} / k T\right)
$$

turns out to be sufficient for recovering all thermodynamic variables with well-established rules and therefore for obtaining the isotherms. However, it can be shown (Yang \& Lee 1952; Griffiths 1972), without solving the $Z_{N}$, that no isotherms can possibly harbor singularities, as are required for showing PT and CP (i.e. a general no-go theorem). The problem is resolved when it is realized that when taking to the thermodynamic limit (TL), i.e. $N \rightarrow \infty$ and $V \rightarrow \infty$ while $N / V$ remains finite, a system in thermo-equilibrium is well-behaved, namely, $F_{\infty}$ (the free energy under TL) and $p_{\infty}$ (the pressure under TL), etc. not only exist but are also smooth except at those points where PTs take place. Exact solutions (Onsager 1944; see also Schultz et al 1964) of the one- and two-dimensional Ising systems show that PTs only occur at systems of at least two dimensions, and for such systems the derived critical exponents reasonably agree with the experimental values. But for more complex systems a method of renormalization group (RS) must be used instead (Kadanoff 1966; Wilson \& Kogut 1974; Fisher 1983, 1998; Batterman 1998). RS provides a recursive technique of rescaling the system so that one can repeatedly collapse blocks of degrees of freedom so that in the end the macroscopic features of PT and CP appear. ${ }^{13}$

3.1. The macroscopic model. As described above, PT and CP are observable phenomena whose patterns can be seen on plotted isotherms of data points; and the isotherms can also be predicted by empirical laws such as the vdW law. Already, the isotherms are idealized in thermodynamics so that PT and CP points are shown as singularities, but the idealizations are minimal. In this sense, there is some similarity between the mechanics of celestial bodies and thermodynamics. As I mentioned earlier, one of the reasons that a relatively precise science of motion for the celestial bodies came long before such a science could even be conceived for the terrestrial bodies is that nature appears to have done the idealization for those who studied the celestial movements. The same seems to be true for thermodynamics in contrast to statistical mechanics. One may nonetheless ask the following question: is it not the case that the truth of all thermodynamic laws depends on a drastic idealization which ignores the molecular nature of bulk matter? Should we not therefore say that all thermodynamic models are among the most (unrealistically) idealized models in science? Comparing to this, whatever idealization one takes for a mechanical model should be far more realistic since it at least recognizes the molecular nature of matter. 
This question brings up an important, and so far neglected, point about idealization. If one is asked to compare a thermodynamic model with an Ising-type model, which should one say is more realistic -- i.e. less idealized? One may say that since ignoring the molecular structure and constructing laws as if fluid is continuous violate our basic metaphysical belief of the molecular nature of bulk matter, thermodynamic models are far less realistic (or far more idealized). But one may also say that since thermodynamic models are far closer to the systems from which empirical generalizations are discovered and/or tested than the Ising-type models are (in fact no empirical generalizations can be draw from an Ising model), the Ising-type models are far less realistic (or far more idealized).

This analysis illustrates the point about the relevance of levels of stability in the phenomena to the evaluation of idealizations. Even though it is one of our fundamental beliefs that matter is made of small particles, the degrees of idealization should not be measured on an absolute scale dictated by this ontological picture. Models of celestial mechanics in which great and complex bodies such as stars are regarded as nearly point masses and models of thermodynamics in which fluid and magnet are regarded as continuous matter are not to be regarded as highly idealized models because they are so close to being the way such systems are presented to us by nature. To us humans, the mid-size macroscopic level is one of the most stable level of natural phenomena on which a whole host of idealized models should be evaluated independently. There are other levels, from the cosmological to that of the elementary particles, on which exist stable phenomena, from which idealized models can be made and evaluated. We should not think that there is an absolute scale. Hence, idealizations are level dependent; and the ones belonging to different levels are not comparable (those in thermodynamics are not any better or worse than those in statistical mechanics). One can only speak of degrees of idealization and make comparison among idealized models within the same level.

3.2. The mean-field models. Serious efforts of idealization arise from the desire to explain why the singularities, among other thermodynamic features of bulk matter, are there. For the PT and CP in fluid, we have (as described above) the vdW model, accompanied with the Maxwell construction (see the Maxwell plateau in Figure 1), and for ferromagnetism, the Weiss model. Once bulk matter is regarded as being made of corpuscular parts, the most extremely idealized model for gas is the ideal gas model, from which Boyle's law can be derived (and thus explained): not only the size of, but also the non-collisional interactions among, the particles are ignored. The model is so impoverished that it cannot represent anything except diluted gases at $T>>T_{c}$. Moreover, the model is so idealized that 
some more drastic idealizations can be added to derive the same Boyle's law, (dramatically simplifying the derivation). To assume that the molecules in a diluted gas all move in straight lines, with the same constant speed, and in directions perpendicular to the walls of the container would be a very unrealistic idealization by anyone's standard, and yet if one subjects the ideal gas model to this additional assumption, one still recovers Boyle's law. ${ }^{14}$ This assumption can of course be dropped and replaced by the famous Maxwell distribution of the molecular velocities, and yet we recover the same Boyle's law.

Here we see yet another interesting aspect of the notion of idealization that is not noticed before: given a law or a theory, the choice of idealized models for it may be underdetermined, which implies that a choice among such models -- i.e. a choice of the idealizations -- cannot be made by comparing their effects on the law or theory alone. In the case of the Maxwell vs. the Bernoulli model of ideal gas, it is clear that the former is chosen because it is more realistic. It is not unlikely that this is one of the two ways by which such cases are resolved. Here is how I see it. Whenever a underdetermined case as described appears, there are two possible ways of making a determination, if one is genuinely called for: either one extends the theory in question to include more phenomena (if it can be done) and the extension breaks the underdetermination, or one makes no extension but evaluates the models against a network of theories and empirical findings.

In the vdW model, the two essential ideal conditions in the ideal gas model is removed. However, neither the exact size nor the exact form of the attraction are specifiable in the model; rather their effects are phenomenologically estimated by the two constants, $b$ and $a$, such that the assumption of the hard-core produces a correction: $v \rightarrow v-b$, and that of the attractive force a correction: $p \rightarrow p+a / v^{2}$, in the equation of state; and the values of the constants are ultimately determined by experiments. From this one can see that the vdW model is not yet a fully microscopic model, although it is microscopic by conception.

As briefly explained earlier, the Weiss model of ferromagnetism consists of dipoles in a solid whose average total spin orientation determines its magnetization. When $T<T_{c}$, the agitation of the dipoles is subdued so much so that a self-induced mean-field of the aligned spins appears with the external field and does not disappear when the external field vanishes. Similar to the dvW model, there is no precise argument for how the model gives rise to ferromagnetism; rather the effect of the model is given phenomenologically by the constant, $\alpha$, in the added term, $\alpha I$, which is in turn determined experimentally. One can see a striking parallelism between the vdW and the Weiss model in the 'ideal gas model $\rightarrow$ vdW model' transition and the 'paramagnetic model $\rightarrow$ Weiss model' 
transition; both involve microscopic but inexact models and the simple insertion (in the equations resulting from the first models) of experimentally determined constants for whatever effects such models may produce. Because the ideal conditions are qualitatively correct and quantitative inexact, there should be plenty of room for further fine-tuning. This type of idealizations agrees well with the traditional theory (see, $[\mathrm{A}]$ above): the evaluation of the mean-field models should depend on whether the fine-tuning eventually works out. To my best knowledge, no such fine-turning were taken seriously.

The first fully microscopic model from which the vdW law is recovered with an explicit microscopic definition of the two constants is perhaps Ornstein's given in 190815. The Hamiltonian of an N-particle system is the sum of two terms: the kinetic and the potential term, which also splits the total partition function of the system accordingly The kinetic part is the same as the one in the Maxwell-Boltzmann theory of ideal gas, and the potential part contains the two-body inter-molecular force term:

$$
\phi\left(\bar{x}_{i j}\right)=\begin{array}{lr}
\infty, & \bar{x}<\delta \\
\phi^{a}\left(\bar{x}_{i j}\right), & \text { otherwise }
\end{array},
$$

where $\bar{x}_{i j}=\left|x_{i}-x_{j}\right|$ is the distance between the two molecules and $\phi^{a}(\bar{x})$ the attractive part of the force which is weak and very long-range. With such a force, the molecules have a hard-core with a radius of $\delta / 2$, and outside the core they attract one another with $\phi^{a}(\bar{x})$. The full derivation should not concern us here (see Emch \& Liu 2001, sect. 11.3); suffice it to point out that the crucial idealization is taken when one replaces the contribution of $\phi^{a}(\bar{x})$ to the partition function by its average:

$$
a=(1 / V) \int d x \phi^{a}(x)
$$

where the integration is taken over the whole system, and $a$ is one of the constants in the vdW law (and $b$ is of course identified with $\delta$, the diameter of the hard-core). Another major model (or a series of models) is the 1-dimensional Kac-Baker model in the early 1960s (cf. Kac 1959; Baker, Jr. 1961). In this model, the idealization (3) is removed and replaced with a stipulation of the exact function of $\phi^{a}(\bar{x})$. This model is much more sophisticated than the Ornstein model: it can derive not 
only the vdW law with more rigor but also the Maxwell plateaus at $T<T_{c}$, which the Ornstein model cannot. 16

One may argue that the Kac-Baker model is obviously better than the Ornstein model, so I am wrong in saying earlier that the micro-models for the vdW law are underdetermined. This is not quite right. As far as recovering the vdW law is concerned, the Kac-Baker model does manage to avoid idealization (3), but it introduces such things as the thermodynamic limit. But is it not true that one can derive the Maxwell plateaus from the Kac-Baker model but not from the Ornstein model? The correct response to this should not be that the Kac-Baker model is a better vdW model, but rather it is probably a better model than any candidate for the vdW model.

This raises the question of which model should be taken as a vdW model. We see from our discussion that the answer to this question is not at all trivial. There is one definite vdW law, which by conception takes the molecular structures of bulk matter seriously. But a model in which the law is true can be a purely thermodynamic model (a rather far-fetched possibility), a model which leaves the microscopic details of the two constants unspecified (the most fitting model, I think, to be called a $\mathrm{vdW}$ model), a model in which the vdW law is derivable with a critical idealization that replaces the attractive part of the inter-molecular force with its average value (the idealization explains why the $\mathrm{vdW}$ is a mean-field model), or a fully microscopic model with some highly unusual conditions of idealization (which seems to have gone beyond the vdW model).

The fully microscopic model from which the Weiss equation of ferromagnetism can be derived is a special case of the Ising-type models, to which I now turn.

3.3. The Ising-type models. If the traditional theory of idealization were right, the development of thermostatistical explanations of PT and CP should have followed the direction of vdW and Weiss models. However, what happened in history was not a pursuit of less idealized laws (or models) along the line of mean-field models -- better values for $a, b$, and $\alpha$ or more constants for more refinement -- but an invention of models to which well-established laws in other fields of physics, such as the laws of (classical or quantum) mechanics and electrodynamics, apply. We have already seen above some fully microscopic models for fluid in the efforts to derive the vdW law. The Isingtype models, as briefly described above, are mostly for ferromagnetism, even though there is a lattice model for fluid, as we shall see in a moment. 
First, let us look at the recovery of the Weiss model from an Ising model. If we follow Weiss in thinking of ferromagnetism as the result of each, say the ith, spin feeling the collective influence of all the other spins and the external field, we can rewrite the Hamiltonian in (1) as follows:

$$
H_{N}=-\sum_{i}\left(\sum_{j \neq i} J_{i j}\left\langle\sigma_{j}\right\rangle+B\right) \sigma_{i}
$$

(where the fluctuation term, $\sum_{j \neq i} J_{i j}\left(\sigma_{j}-\left\langle\sigma_{j}\right\rangle\right) \approx 0$ ), which differs from a paramagnet's Hamiltonian, $H_{N}=-\sum_{i} B \sigma_{i}$, by a simple substitution of $B \rightarrow B+\sum_{j \neq i} J_{i j}\left\langle\sigma_{i}\right\rangle$ (where, $J_{0}=\sum_{j \neq i} J_{i j}$ ). This is the

Weiss model, with its substitution scheme: $B \rightarrow B+\alpha I$, and here we see the meaning of the coefficient, $\alpha$, which is the sum of the coupling constants between any two sites. Similar to the Ornstein model for recovering the vdW equation of state, the Ising model shows how exactly the mean-field appears in the Weiss model; and also like the Ornstein model, there is no precise way of calculating $I=\left\langle\sigma_{j}\right\rangle$ or $\alpha=J_{0}$ either rigorously or in a controlled approximation for all Ising-type models.

We now turn to the discussion of the idealizations involved. The variety of the Ising-type models is generated by the number of dimensions, the type of site occupancy, and the type of interactions. Some models are simple to use but unrealistic, while the more realistic ones are notoriously difficult to handle: the simplest model is perhaps a 1-dimensional string of finite length on which spin sites are located at equal distance; and each site takes only one of two spin states -- up or down -- and interacts only with its nearest neighbors. And the most complex is perhaps a 3dimensional honeycomb shape lattice of equal distanced sites on which two spin values are possible, and the interactions are not only among the nearest neighbors. ${ }^{17}$ When I mentioned the derivation of the Weiss model above, I deliberately avoided specifying which among these Ising-type models must

be used. To recover the equation and leave the value of $J_{0}=\sum_{j \neq i} J_{i j}$ and $\left\langle\sigma_{j}\right\rangle$ in (4) to the experiments, one can use any of the Ising models.

It should be obvious by now that from the mean-field models to the Ising-type ones a fundamental shift has taken place in terms of the quality or the degrees of idealization. A sense of this may be had if we ask the question: which type of models is a better idealized one? If this question is put to us concerning instead the ideal gas model and the vdW model, we would have no difficulty in giving a definite answer. The ideal conditions for the latter is not only more realistic than the former 
but also produces via the vdW law a more approximate description of thermo-systems than the former can produce via Boyle's law. The same cannot be said unambiguously for the previous pair -between the mean-field and the Ising-type models.

For the ferromagnet, we have the standard Ising-type models, as described earlier; but for fluid, the Ising-type model is the lattice gas (cf. Yang 1952), which is built out of an Ising magnet by a simple transformation. For this, any Ising lattice will do: instead of having on each site a spin of up or down, we have the site as 'occupied (by a molecule)' or 'not occupied.' Hence, we switch from a configuration of spin orientation pattern on a lattice to that of occupation pattern on a lattice. In terms of random variables, where $\sigma$ is for spin and $n$ for the occupation number of molecules, the switch is as follows:

$$
\sigma: i \in \Lambda \text { a } \sigma_{i} \in\{+1,-1\} \Rightarrow n: i \in \Lambda \text { a } n_{i} \in\{0,1\}
$$

Despite its rather artificial appearance, the lattice gas model can do a great deal (as briefly noted above). And with some modification, such as in the Kac-Baker model, one can even use it to rigorously recover the dvW law. It is especially a suitable model for solutions and alloys.

To truly answer the question of which type of idealized models are better, we need to consider several aspects of this comparison. First, the mean-field models are more for the discovery of laws than for the application, whereas the Ising-type models are the opposite. I say 'more for' rather than 'definitely for' because some known laws are used -- even if implicitly -- in the mean-field models. However, the construction of mean-field models, such as the vdW and the Weiss model, is mainly for the discovery of semi-empirical laws which account for the phenomena. The laws are derivable neither from purely thermodynamic principles and/or laws nor from statistical mechanical laws within the models; therefore, they are new laws discovered in the corresponding mean-field models. The Ising-type models are also constructed to account for the phenomena -- especially PT and CP, but the aim is to see to it that the account follows from the (heavily tailored) application of laws in mechanics and electrodynamics in the models.

Second, idealizations which make the mean-field models are certainly not as exact as the ones which make the Ising-type models, but they are by no means less realistic. A judgment on which are more realistic is difficult because the difference is subtle. The key idea of mean-field models is precisely to use the 'mean-field', namely, to use the averages of what are in reality so complex as to defy any precise knowledge. One of the key assumption behind this use of average is of course the 
well-supported belief that the deviations are negligibly small. On the other hand, the key idea of the Ising-type model is precisely to use the 'lattice', a rigid structure with all the particles separated by equal, or multiples of equal, distances. Such models (or their idealizations) are clearly less realistic than the mean-field models (or their idealizations). Perhaps one can say that the mean-field models are as inexact as the Ising-type models are unrealistic; but this is not quite true, and a simple discrepancy of the two shows why. As I mentioned earlier, while the Ising-type models predict the right critical exponents, the mean-field models get the wrong values. This must mean that taking the averages in the ways in which the mean-field models take them is too crude for characterizing the fluid or the ferromagnet near the critical point. Hence, the mean-field models are simply not acceptable models regardless of whether they are more realistic than the Ising-type models.

Third, despite their theoretical power, the Ising-type models are simply not realistic. We can see this by a simple argument. There is a no-go theorem for all Ising-type models which says that there is no possibility for any PT (and hence no $\mathrm{CP}$ ) in any such model systems with finite extensions. To recover PT, one must take the thermodynamic limit, as briefly described earlier. Once that is taken, it is found that there is no PT in a 1-dimensional string of nearest-neighbor interactions or interactions of similar short-ranges, and one needs at least a 2-dimensional system for that to emerge. Now, taken literally, the no-go theorem should be a simple refutation of the Isingtype models, which should naturally lead to a rejection of the idealizations (as unrealistic). Literally speaking, most, if not all, PTs take place in finite systems, and yet when modeled by Ising models as their micro-structures, none of them can harbor PTs. The best interpretation of this situation is I believe the following. A ferromagnet, for instance, is neither an Ising lattice nor an infinite system; but there are good theoretical reasons to idealized it into a model of infinite Ising-type lattice, and among the reasons, I am certain, is that this is a, if not the, model in which a relatively simple adaptation of laws in mechanics and electromagnetism can be carried out to rigorously derive PT and CP. As is argued elsewhere (cf. Liu 1999b), the thermodynamic limit is in fact a species of idealization that, in different guises, is widely used in physical sciences; the most common variation of which is to assume an otherwise porous and malleable body solid and rigid ('filling in' the gaps by an infinite number of parts). It seems that by introducing two radically unrealistic idealizations -- the Ising lattice and the thermodynamic limit -- one is able to do better than what one can with the less idealized mean-field models. 
4. Conclusion. Let me now summarize the main points of arguments and observations in this paper and point out some remaining issues for future investigations. Approximations in general cannot be evaluated on a single (a few) simple measure(s). This is so not only because there are laws of limits in nature but also because laws and regularities attribute likelihoods to possible states that the proposed universal measures cannot handle. A single (a few) simple measure(s) for idealization, such as approximation approbation, is (are) also unlikely to work because there are not one but two dimensions in the measure of 'closeness' (to a true law) of an idealized (or approximate) law: how much it resembles the facts and how much it resembles the form (or 'shape') of the regularity (which also applies to counterfactual cases). It seems rather hopeless to try to come up with a single measure of the closeness of forms in approximate laws; and even if such a measure can be had, one has to deal with the balance between it and the closeness to facts. Moreover, idealizations -- and idealized models as their products -- are not just for idealized laws. If some acts of idealizations are used to detach component systems or processes from joints of nature, the result may well be the discovery or application of true laws. Indeed, none of the theoretical laws at the microscopic level can be discovered or applied without idealized models, and yet there is no reason to believe that they must therefore be idealized (or approximate) because of the use of such models.

We see three different levels of idealized models in the theory of thermostatistical physics. The thermodynamic level is the closest to direct experiments and therefore needs the least idealization, except the level itself is sealed off by an almost metaphysical idealization which ignores the molecular nature of bulk matter. The result of this idealization is thermodynamic laws and state equations that describe fluctuationless processes. The semi-microscopic level sees models that acknowledge the molecular structures but pack the information into average quantities that appear in the phenomenological laws with empirical constants whose values are determined experimentally. At the fully microscopic level we find models with their micro-structures fully -- if somewhat artificially -specified. Just when one might think that the idealizations which make the Ising-type models may deprive of them the possibility of explaining PT and CP (i.e. the no-go theorem), another idealization -- taking the thermodynamic limit -- comes to the rescue; and together not only PT is recovered but with the help of renormalization group technique one can also make accurate calculations to reveal the details of CP regions.

This paper mainly discussed those idealized models which are intended for the discovery or the application or both of laws of nature. Some such laws are true but some are only true in the models -- which I call idealized or approximate. Now, are thermodynamic laws on the first level true 
or approximate laws? This seemingly innocent question is actually difficult to answer. Judging from the enormous effort by the physicists and mathematicians in trying to 'recover' those laws from statistical mechanics, one would conclude that they are true laws; but the very effort clearly reveals that from a microscopic perspective those laws must admit exceptions, however unlikely such excepts are. ${ }^{18}$ It is fairly obvious that the laws in the mean-field models (at the second level) are meant to be taken as improvable approximate laws. But the true laws for thermo-phenomena turn out not to be greatly improved mean-field laws, but true laws from other areas of physics which apply in highly unrealistically idealized models, such as the Ising-type models.

This paper has barely scratched the surface of the notions of idealization and approximation. Many deeper issues or possibilities remain. Must ideal conditions be physically possible? The answer seems to be 'Yes', but what do we do then with infinite volumes and continuous edges? I argued for the conclusion that some idealized models give true laws but some only approximate ones. ${ }^{19}$ It seems that going down one level always gives one cases of exceptions for the laws obtained as universal at the upper level. But this is a very preliminary speculation. One may simply ask the following question: why should Boyle's law be false -- though somewhat approximate -while the law of inertia be true?. Very roughly put, Boyle's law is true for a diluted tank of sizeless and forceless particles while the law of inertia is true for a forceless region. Shouldn't they have the same truth value? 


\section{References}

Apostel, L. (1961). “Towards the Formal Study of Models in the non-Formal Sciences.” The Concept and the Role of the Model in Mathematics and Natural and Social Sciences. Ed. Freudenthal, H. Synthese Library. Dordrecht, D. Reidel Publishing Company. pp. 1-37.

Armstrong, D. M. (1978). Universals and Scientific Realism, vol. 2: A Theory of Universals. Cambridge: Cambridge University Press.

Armstrong, D. M. (1983). What Is a Law of Nature? Cambridge, Cambridge University Press. Baker, Jr. G.A. (1961). “One-dimensional Order-Disorder Model Which Approaches Phase Transition." Physical Reviews. 122: 1477-1484.

Barr, W. F. (1971). “A Syntactic and Semantic Analysis of Idealizations in Science.” Philosophy of Science 38: 258-272.

Barr, W. F. (1974). "A Pragmatic Analysis of Idealizations in Physics." Philosophy of Science 41: 48-64.

Batterman, R.W. (1998). "Why Equilibrium Statistical Mechanics Works: Universality and the Renormalization Group." Philosophy of Science. 65: 183-208.

Braithwaite, R. B. (1962). Models in the Empirical Sciences. Logic, Methodology and Philosophy of Science: Proceedings of the 1960 International Congress. Eds. E. Nagel, P. Suppes and A. Tarski. Stanford, Stanford University Press. pp. 224-231.

Callender, C. (2001). "Taking Thermodynamics (Too) Seriously." forthcoming in Studies in History and Philosophy of Modern Physics.

Carroll, J.W. (1994). Laws of Nature. Cambridge, Cambridge University Press.

Cartwright, N. (1983). How the Laws of Physics Lie. Oxford, Clarendon Press.

Cartwright, N. (1989). Nature's Capacities and Their Measurement. Oxford, Clarendon Press.

Cartwright, N. (1997). "Models: the Blueprints for Laws." Philosophy of Science. 64: S292-S303.

Cartwright, N. (1999). "Models and the limits of theory: quantum Hamiltonians and the BCS models of superconductivity." in Morgan \& Morrison 1999, pp. 241-281.

Creary, L. (1981). "Causal Explanations and the Reality of Natural Component Forces.” Pacific Philosophical Quarterly. 62: 148-157.

Dretske, F. (1977). "Laws of Nature.” Philosophy of Science 44: 248-268.

Emch, G.G. (1971). Advanced Statistical Mechanics. (Lecture Notes). Preprint, Rochester, NY.

Emch, G.G. and Liu, C. (2001).The Logic of Thermostatistical Physics. Berlin, Springer-Verlag (forthcoming).

Fisher, M. (1974). "The Renormalization Group in the Theory of Critical Behavior." Review of Modern Physics. 46: 597-616.

Fisher, M. (1983). Scaling, Universality and Renormalization Group Theory. Critical Phenomena: Proceedings of the Summer School, the University of Stellenbosch, South Africa, January 18-29, 1982. Ed. F.J.W. Hahne. Berlin, Springer-Verlag, 1-139.

Fisher, M. (1998). "Renormalization Group Theory: Its Basis and Formulation in Statistical Physics." Review of Modern Physics. 70: 653-681.

Giere, R. (1988). Explaining Science: A Cognitive Approach. Chicago, University of Chicago Press.

Goldenfeld, N. (1992), Lectures on Phase Transitions and the Renormalization Group. Reading, MA, Addison-Wesley.

Griffiths, R. B. (1972), Rigorous Results and Theorems. Phase Transitions and Critical Phenomena. Eds. C. Domb and M. E. Green. New York, Academic Press, 8-109. 
Herfel, W., W. Krajewski, I. Niiniluoto and R. Wójcicki, Eds. (1995). Theories and Models in Scientific Processes. (Poznan Studies, vol. 44). Amsterdam, Rodopi.

Hesse, M. (1970). Models and Analogies in Science. Notre Dame, University of Notre Dame Press. Hilpinen, R. (1976). Approximate Truth and Truthlikeness. Formal Methods in the Methodology of Empirical Sciences Eds. M. Przlecki, K. Szanianwski and R. Wojicki. Dordrecht, Reidel, 1942.

Horgan, J. (1994). "Icon and Bild: A Note on the Analogical Structure of Models - The Role of Models in Experiment and Theory." The British Journal for the Philosophy of Science. 45: 599-604.

Kac, M. (1959). “On the Partition Function of a One-dimensional Gas.” Physics of Fluids. 2: 8-12.

Kadanoff, L.P. (1966). "Scaling Laws for Ising Models near Tc." Physics 2: 263-272.

Kline, A. and Matheson, C. (1986). "How the Laws of Physics Don't Even Fib?" PSA 1986 Eds. P.D. Asquith et al. East Lansing, Philosophy of Science Association. 1: 336-350

Krajewski, W. (1977). Correspondence Principle and the Growth of Knowledge. Dordrecht, Reidel. Kyburg, H.E. Jr. (1990). Science and Reason. Oxford: Oxford University.

Laymon, R. (1980). Idealization, Explanation, and Confirmation. PSA 1980 Eds. P. D. Asquith and R. N. Giere. East Lansing, Philosophy of Science Association. 1: 336-350.

Laymon, R. (1985). Idealizations and the Testing of Theories by Experimentation. Observation. Experiment and Hypothesis in Modern Physical Science Eds. P. Achinstein and O. Hannaway. Cambridge, MA, MIT Press. 147-173.

Laymon, R. (1989). "Cartwright and the Lying Law of Physics.” Journal of Philosophy 86: 353372.

Lewis, D. (1973). Counterfactuals. Cambridge, MA, Harvard University Press.

Lewis, D. (1983). "New Works for a Theory of Universals." Australasian Journal of Philosophy. 61: 343-371.

Lewis, D. (1989). On the Plurality of Worlds. Oxford, Blackwell.

Liu, C. (1997). "Theories and Models I: the Semantic View Revisited." International Studies in the Philosophy of Science 11: 147-164.

Liu, C. (1999a). "Approximation, Idealization, and the Laws of Nature." Synthese. 118: 229-256.

Liu, C. (1999b). "Explaining the Emergence of Cooperative Phenomena." Philosophy of Science. 66(S): S92-S106.

Liu, C. (2001a). (in press) "Infinite Systems in SM Explanations: Thermodynamic Limit, Renormalization (semi-) Groups, and Irreversibility." Philosophy of Science. 68.

Liu, C. (2001b). " Laws and Models in a Theory of Idealization." (Under review with Synthese.)

McMullin, E. (1968). What Do Physical Models Tell Us? Logic, Methodology and Philosophy of Science III. Eds. B. van Rootselaar and J. F. Staal. Amsterdam, North Holland. 392.

McMullin, E. (1985). "Galilean Idealization." Studies in the History and Philosophy of Science 16: 247-273.

Marquis, J.-P. (1991). “Approximations and Truth Space.” Journal of Philosophical Logic 20: 375401.

Maxwell, J.C. (1875). "On the Dynamical Evidence of the Molecular Constitution of Bodies." Nature 11: 357, 374, or The Scientific Papers of James Clark Maxwell (Dover, 1965), vol. 2, 418

Mill, J.S. (1874). A System of Logic. 8th ed. New York, Harper and Brothers.

Morgan, M.S. and Morrison, M. eds. (1999). Models as Mediators: Perspectives on Natural and Social Science. Cambridge: Cambridge University Press. 
Morrison, M. (1997). "Physical Models and Biological Contexts." Philosophy of Science. 64: S315-S324.

Morrison, M. (1999). "Models as autonomous agents." in Morgan \& Morrison 1999, pp. 38-65.

Niiniluoto, I. (1984). Is Science Progressive. Dordrecht, Reidel.

Niiniluoto, I. (1986). Theories, Approximations and Idealizations. Logic, Methodology and Philosophy of Science Eds. R. B. Marcus and e. al. Amsterdam, North Holland. 255-289.

Niiniluoto, I. (1987). Truthlikeness. Dordrecht, Reidel.

Niiniluoto, I. (1998). "Verisimilitude: The Third Period." The British Journal for the Philosophy of Science. 49: 1-29.

Nowak, L. (1972). "Laws of Science, Theories, Measurement." Philosophy of Science 39: 533-548.

Onsager, L. (1944), "Crystal Statistics. I. A Two-Dimensional Model with an Order-Disorder Transition." Physical Review 65: 117-149.

Ramsey, J. L. (1992). Towards an Expanded Epistemology for Approximations. PSA 1992 vol. 1, Eds. D. Hull, M. Forbes and K Okruhlik. East Lansing, Michigan, Philosophy of Science Association. 154-164.

Redhead, M. (1980). "Models in Physics." The British Journal for the Philosophy of Science. 31: 145-163.

Rosenkrantz, R. (1980). “Measuring Truthlikeness.” Synthese 45: 463-488.

Schultz, T.D., Mattis, D.C. and Lieb, E.H. (1964). "Two-dimensional Ising Model as a Soluble Problem of Many Fermions." Review of Modern Physics 36: 856-871.

Schwartz, R. J. (1978). "Idealization and Approximations in Physics." Philosophy of Science 45: 595-603.

Sklar, L. (1993). Physics and Chance. Cambridge: Cambridge University Press.

Stanley, H. E. (1971). Introduction to Phase Transitions and Critical Phenomena. Oxford: Clarendon Press.

Suárez, M. (1999). "The role of models in the application of scientific theories: epistemological implications." in Morgan \& Morrison 1999, pp. 168-196.

Suppe, F. (1989). The Semantic Conception of Theories and Scientific Realism. Urbana, Ill, University of Illinois Press.

Toda, M., R. Kubo and N. Saitô (1983). Statistical Physics I: Equilibrium Statistical Mechanics. Berlin: Springer-Verlag.

van Fraassen, B. (1989). Laws and Symmetry. Oxford, Clarendon Press.

Weston, T. (1987). “Approximate Truth.” Journal of Philosophical Logic 16: 203-227.

Weston, T. (1992). “Approximate Truth and Scientific Realism.” Philosophy of Science 59: 53-74.

Wilson, Kenneth G. and J. Kogut (1974), "The Renormalization Group and the $\varepsilon$ Expansion." Physics Report. 12C: 75-197.

Wimsatt, W.C. (1987). "False Models as Means to Truer Theories," in M. Nitecki \& A. Hoffman (eds.) Neutral Models in Biology. Oxford, Oxford University Press, 23-55.

Wimsatt, W.C. (1994). "The Ontology of Complex Systems: Levels, Perspectives, and Causal Thickets," in M. Matthen \& R. X. Ware (eds.) Biology and Society: Reflections on Methodology. Canadian Journal of Philosophy, supplementary vol. 20: 207-274.

Yang, C. N. and T. D. Lee (1952), "Statistical Theory of Equations of State and Phase Transitions I. Theory of Condensation." Physical Review 87: 404-409.

Yang, C. N. (1952), "The Spontaneous Magnetization of a Two-Dimensional Ising Model." $\underline{\text { Physical }}$ Review 85: 808. 


\section{NOTES}

$\dagger$ I would like to thank Gérard Emch for numerous discussions throughout this project. I would also like to thank four anonymous reviewers from NSF for comments on an earlier version of this paper. This paper is supported in part by an NSF grant: SES-9910889.

${ }^{1}$ Since there is no danger of mistaking a law-sentence for a law in the context of our discussion here, I shall often use the term 'law' for both and let the contexts tell which is meant. This saves me from writing such odd phrases as 'Boyle's law-sentence' rather than 'Boyle's law', which in some context refers to the statement of the law and in others the law itself.

2 It is far beyond the scope of this paper to give an adequate account of Niiniluoto's theory of approximation and verisimilitude. For a recent survey, see Niiniluoto 1998.

3 For other similar approaches, see Krajewski 1977; also Schwartz 1978; Rosenkrantz 1980; Niiniluoto 1987; Cartwright 1989, ch.5; Kyburg 1990, ch.10.

${ }^{4}$ For other criticisms of the traditional theory, see Ramsey 1992.

5 One should note that most laws in physics and many laws in science are such that the F's and G's are property variables -- magnitudes or quantities -- which are really classes or bundles of properties. For such laws, the form may be better given as, $N[P, Q \mid Q=f(P)]$, which means that for every value of $\mathrm{P}$ and $\mathrm{Q}$, it is naturally necessary that all P's are $\mathrm{Q}$ 's; or more accurately, given $\mathrm{D}_{\mathrm{p}}$ and $\mathrm{R}_{\mathrm{q}}$ as the domain for P's values and the range for Q's values, for every value of $\mathrm{P}^{\mathrm{i}} \in \mathrm{D}_{\mathrm{p}}$, there is a $\mathrm{Qj} \in \mathrm{R}_{\mathrm{q}}$, such that $\left.\forall \mathrm{xN}\left[\mathrm{Pi}^{\mathrm{i}} \mathrm{x}\right), \mathrm{Qj}(\mathrm{x})\right]$.

6 The natural necessity is the same as physical necessity for a physicalist; but for a non-physicalist, the natural is broader than the physical. Psychophysical laws, if exist, are natural but not physical.

7 Idealized models may also be used for testing a law or a theory, but this role is quite different from the other two roles -- discovery and application -- because if by 'testing' we mean checking the law in question whether it is consistent with a set of other laws to which it should belong, some models certainly serve this logical function; but if by that we mean checking -- i.e. doing experiments and interpreting data -- whether the law is actually true, it must be possible for the idealized models to be approximately realized. This is a severe demand that most highly theoretical models, e.g. in the microscopic or cosmological realms, cannot meet. Therefore, many theoretical laws are not directly tested in their own models but rather indirectly tested in models that are close to the phenomena.

8 For early discussions of models, see Apostel 1961; Braithwaite 1962; McMullin 1968; Hesse 1970; for alternative views, see Redhead 1980, Horgan 1994; and for a review, see Liu 1997. 
${ }^{9}$ For the details of this argument, see Liu $2001 b$.

10 For more on this, see also Creary 1981; Kline \& Matheson 1986; Laymon 1989.

11 One may worry about the following possibility: we have not discovered a single law in science and we may never discover any since all of our known 'laws' and all those we will in fact discover are only approximate ones. If so, the distinction between approximate laws and true laws in idealized models would be of no practical meaning. This is however not quite true. Perhaps none of the laws we regard true is in fact true, but those we do have reasons to believe as true will not be false because of the idealized models we use to either discover or apply them. The current law of gravitation may only be approximately true, but it will not turn out to be false because we studied it in separation from other force fields, such as electromagnetism. This is why I can use the Newton's theory of gravity and Maxwell's theory of electromagnetism to illustrate how laws which hold in idealized models may hold in general. Both are false theories but not false because we have studied them separately.

12 For instance, as shown in Figure 1, the liquid-gas PT of a fluid below the critical temperature is bounded by two (singular) points in the $\mathrm{pV}$ isotherm, across which 'rates' such as $[\partial \mathrm{p} / \partial \mathrm{V}] \mathrm{T}$ are not continuous.

13 See (Liu 1999b, 2001a) for more detailed discussion.

14 D. Bernoulli in fact did the calculation under such a model with a few more ideal conditions in 1738, see Emch \& Liu, 2001, sect. 3.2.

15 The original derivation is given by L.S. Ornstein in his dissertation (in Dutch) in Leiden with a title whose English translation reads: Application of the statistical mechanics of Gibbs to molecular theoretical questions. For its contemporary rendering, see Emch \& Liu 2001, sect. 11.3.

16 The derivation is more rigorous at least in the sense that by replacing the attractive force, $\phi^{a}(\bar{x})$, with its average, (3) is not an approximation that one can estimate in a controllable manner, i.e. being able to produce a numerical estimate of whatever degree of approximation. However, it is only a 1dimensional model and involves some mathematical maneuvers which seems to be extreme measures of idealization: one needs to take the thermodynamic limit in order to derive the vdW law in this model, and one has to put the quantity,

$$
A^{-1} \int_{o}^{\infty} x \phi^{a}(x) d x
$$

to zero while maintaining that 


$$
A=\int_{o}^{\infty} \phi^{a}(x) d x
$$

be finite. I shall discuss in detail the nature of thermodynamic limit later. The extension to higher dimensions seems to be conceptually transparent, but technically it is by no means straightforward. In this aspect, the Kac-Baker model is very much like the Ising-type models.

17 There are more complex models. One must note that the Ising-type models are really classical models because the spin variable on each site is not treated as a quantum operator as it should in quantum theory but rather as a two-valued classical variable. The corresponding quantum models are know as the Heisenberg models, on whose sites sit particles of quantum spins, namely they take all the eigenvalues of a quantum spin as possible values.

18 For an in-depth discussion of the whole issue, see Callender 2001.

19 More accurately I should say that I argued, mostly elsewhere (Liu 2001b), that idealized models can produce universally true laws. I have not argued that idealized models can also produce approximate laws, which in view of laws such as Boyle's and Weiss's I take to be fairly obvious. 